\title{
openheart Efficacy and safety of dabigatran versus warfarin from the RE-LY trial
}

\begin{abstract}
Alvaro Avezum, ${ }^{1}$ Gustavo Bernardes de Figueiredo Oliveira, ${ }^{1}$ Rafael Diaz, ${ }^{2}$ Jesus Antonio Gonzalez Hermosillo, ${ }^{3}$ Jonas Oldgren, ${ }^{4}$ Ernesto Ferreiros Ripoll, ${ }^{5}$ Herbert Noack, ${ }^{5}$ Leopoldo Soares Piegas, ${ }^{6}$ Stuart J Connolly, ${ }^{7}$ on behalf of the RELY Investigators
\end{abstract}

\begin{abstract}
- Additional material is published online only. To view please visit the journal online (http://dx.doi.org/10.1136/ openhrt-2018-000800).
\end{abstract}

To cite: Avezum A, Oliveira GBdF, Diaz R, et al. Efficacy and safety of dabigatran versus warfarin from the RE-LY trial. Open Heart 2018;5:e000800. doi:10.1136/ openhrt-2018-000800

Received 27 February 2018 Revised 15 April 2018 Accepted 1 May 2018

Check for updates

'Dante Pazzanese Institute of Cardiology, São Paul, Brazil ${ }^{2}$ Estudios Clinicos Latinoamerica, Rosario, Argentina

${ }^{3}$ Instituto Nacional de Cardiologia Ignacio Chavez, Rosario, Argentina

${ }^{4}$ Uppsala Clinical Research Center and Department of Medical Sciences, Uppsala University, Uppsala, Sweden ${ }^{5}$ Boehringer Ingelheim Pharma $\mathrm{GmbH}$ and $\mathrm{Co}$. KG, Ingelheim am Rhein, Germany

${ }^{6}$ Hospital do Coração, São Paulo, Brazil

${ }^{7}$ Population Health Research Institute, McMaster University and Hamilton Health Sciences, Hamilton, Ontario, Canada

Correspondence to Dr Alvaro Avezum; avezum@ yahoo.com

\section{ABSTRACT}

Background Current data for atrial fibrillation (AF) and stroke are predominantly derived from North American and European patients. Although the burden of AF is high in Latin America (LA), little is known about current management of $\mathrm{AF}$ in the region.

Methods We aimed to assess the consistency of efficacy and safety outcomes associated with dabigatran etexilate (DE) versus warfarin in patients with AF in LA from the RE-LY (Randomised Evaluation of Long-Term Anticoagulant Therapy) trial. Data from 956 LA patients and 17157 non-LA patients were included in this analysis. $\chi^{2}$ test and Cox proportional regression analysis were performed. The primary efficacy outcome included all strokes or systemic embolism (SE). Main safety outcome was major bleeding. Results LA patients were more often female, had higher proportion of permanent AF and lower creatinine clearance, among other characteristics. Vitamin $\mathrm{K}$ antagonist use at randomisation and time in therapeutic range were lower in LA than in non-LA patients $(44 \%$ vs $63 \%, \mathrm{p}<0.001$; and $61.3 \pm 22.6 \%$ vs $64.6 \pm 19.6 \%$, $\mathrm{p}=0.015$, respectively). Efficacy endpoints were $0.91 \%$ versus $1.68 \%$ for $D E 150 \mathrm{mg}$ twice daily versus warfarin, respectively. Stroke/SE risk was lower in LA patients treated with DE $150 \mathrm{mg}$ twice daily compared with warfarin, although not significant (HR 0.54; 95\% Cl 0.18 to 1.62). The annual stroke/SE rates for DE $110 \mathrm{mg}$ twice daily versus warfarin were 1.82 versus 1.68 , also not significantly different (HR 1.09; $\mathrm{Cl} 0.44$ to 2.67). There were no treatment-by-region interactions for either dose of DE on efficacy and safety outcomes.

Conclusion Despite differences in the clinical profile and AF management, the efficacy and safety benefits of dabigatran over warfarin in LA patients relative to non-LA patients are consistent with those observed in the main RE-LY trial.

\section{INTRODUCTION}

Atrial fibrillation (AF) is responsible for $\sim 15 \%-20 \%$ of all strokes. ${ }^{12}$ AF occurs in $1 \%-2 \%$ of the population and its prevalence increases with age. ${ }^{3}$ Most of the epidemiological data available for AF and related stroke predominantly are derived from patients from North America and Europe. ${ }^{4}$ Although the burden of AF is high in Latin America (LA), for instance, in Brazil, 1.5 million

\section{Key questions}

What is already known about this subject?

- Non-vitamin K antagonist oral anticoagulants (NOACs) are safer and more effective than warfarin in the management of patients with atrial fibrillation (AF).

- Globally, scientific evidence from clinical trials is compelling for NOACs use among AF patients.

- Several NOACs have been adequately tested in large randomised clinical trials; nonetheless, most data are derived from patients enrolled from high-income countries.

What does this study add?

- Efficacy and safety profile of dabigatran versus warfarin among patients from low-income and middle-income countries from Latin America reassuring broad NOAC applicability.

- Consistency of results as observed in the overall findings from the main RE-LY (Randomised Evaluation of Long-Term Anticoagulant Therapy) study.

- Potential change in regional practice (Latin America) towards improvement in the stroke and systemic embolism prevention in patients with AF.

How might this impact on clinical practice?

- Reassurance of consistency of efficacy/safety profile of dabigatran might lead physicians to greater use of NOAC in the management of patients with AF

- Due to the large stroke burden in Latin America, this information could enhance the implementation of more effective and safer treatments (NOACs) to fight stroke and related death or disabling outcomes.

- Deliver of care by progressive and broader use of safer and more effective anticoagulants (NOACs) along with simplicity of its use in the region would be instrumental for adherence to local, regional and international guidelines.

people have $\mathrm{AF},{ }^{5}$ little is known about current management of $\mathrm{AF}$ and related stroke in developing countries. The incidence of first and recurrent strokes, intracranial and subarachnoid haemorrhages is higher in LA than in populations from North America or Europe, including non-Hispanic whites. This 
increased risk of stroke extends to individuals with $\mathrm{AF}$ from LA. ${ }^{6-9}$ For instance, WHO estimated that nearly 2.0 million people had survived a stroke in LA in 2004, and about $25 \%$ of them experienced a first episode of stroke. Recent epidemiological data suggest a rapid increase in the incidence of strokes over the last two decades, which represents a trend seen in many Latin American nations. Furthermore, some data have shown that there is a relatively higher rate of haemorrhagic stroke in these LA countries compared with high-income nations (26\% vs $9 \%)$.

Therefore, the optimal management of AF with appropriate use of oral anticoagulant therapy is of great relevance, particularly in LA. Traditionally, vitamin K antagonists (VKAs) and aspirin have been prescribed to reduce the risk of stroke in patients with AF. The use, management of care and time in therapeutic range (TTR) as an indicator of quality of oral anticoagulation with VKAs are reported to be suboptimal in South American or Latin American groups. ${ }^{10-12}$ Nevertheless, goodquality management of AF with VKAs is still possible in deprived South American populations. ${ }^{13}$

Several non-VKA oral anticoagulants (NOACs) have been developed and tested in randomised clinical trials as alternatives to warfarin. Dabigatran etexilate (DE) is an oral direct thrombin inhibitor, and rivaroxaban, apixaban and edoxaban are direct factor Xa inhibitors. ${ }^{14-17}$ The RE-LY (Randomised Evaluation of Long-Term Anticoagulant Therapy) trial showed that dabigatran $150 \mathrm{mg}$ twice daily was more effective than warfarin for the prevention of stroke and systemic embolism (SE) in patients with $\mathrm{AF}$, with similar rates of major bleeding. ${ }^{14}$ In addition, dabigatran given at a dose of $110 \mathrm{mg}$ twice daily was associated with similar rates of stroke and SE as compared with warfarin, as well as lower rates of major bleeding.

Patients in the RE-LY trial were recruited from a wide range of countries in terms of education, culture, income, healthcare systems and access to medications. The majority of them were enrolled in Western Europe and in the USA/Canada. ${ }^{14} 18$ Because of this broad geographical representation, various patient characteristics and expected practice variation, regional analyses offer a unique opportunity to help further delineate the effects of dabigatran versus warfarin on patients from different geographical areas, clinical profiles and access to anticoagulant therapies. For instance, previous subanalyses of RE-LY data focusing on Japanese ${ }^{18}$ and Asian populations ${ }^{19}$ have observed the following: higher haemorrhagic stroke rates on warfarin in Asians versus non-Asians, despite similar blood pressure, younger age and lower international normalised ratio values; significant reduction in haemorrhagic strokes by $\mathrm{DE}$ in both Asians and non-Asians, demonstrating consistent benefit of DE versus warfarin across Asian and non-Asian subgroups.

Since there was a relatively large cohort of randomised patients $(n=956)$ from LA in the RE-LY trial and the high burden of AF and related strokes in this region, we aimed to assess geographical differences in terms of efficacy and safety outcomes associated with dabigatran versus warfarin in patients with $\mathrm{AF}$, specifically comparing patient data from LA and non-LA countries to evaluate potential interaction between treatment and region.

\section{METHODS \\ Design}

This analysis was based on the phase III, prospective, randomised, open-label multinational RE-LY trial, in which 18113 patients (951 centres in 44 countries) were randomised to two fixed doses of dabigatran (110 or $150 \mathrm{mg}$ twice daily) or warfarin (target international normalised ratio (INR): 2.0-3.0) with a median follow-up of 2 years. ${ }^{1420}$

\section{Population}

Patients were considered eligible if they had a documented diagnosis of non-valvular AF (paroxysmal, persistent and permanent) and at least one additional risk factor for stroke, which included previous ischaemic stroke or transient ischaemic attack, left ventricular ejection fraction $<40 \%$, New York Heart Association class II-IV heart failure and age $\geq 75$ years (or age $\geq 65$ years plus diabetes mellitus, coronary artery disease or hypertension). Reasons for exclusion were the presence of severe heart valve disorder, stroke within 14 days or severe stroke within 6 months before screening, any condition that increased the risk of haemorrhage, creatinine clearance $<30 \mathrm{~mL} / \mathrm{min}$, active liver disease, or pregnancy.

LA countries included Argentina, Brazil, Colombia, Mexico and Peru. All remaining countries included in the entire trial were considered to be non-LA countries.

\section{Study outcomes}

The primary outcome was the occurrence of stroke (ischaemic and haemorrhagic) or non-central nervous system systemic embolism. Other efficacy outcomes included myocardial infarction and death from any cause. The primary safety outcome was major bleeding (reduction in haemoglobin level of $\geq 2 \mathrm{~g} / \mathrm{L}$, transfusion of $\geq 2$ units (U) of blood or symptomatic bleeding in a critical area or organ). Other safety outcomes were life-threatening bleeding (fatal bleeding, symptomatic intracranial haemorrhage (ICH), bleeding with a decrease in haemoglobin level of $\geq 5 \mathrm{~g} / \mathrm{L}$ or bleeding requiring transfusion of $\geq 4 \mathrm{U}$ of blood, inotropic agents or necessitating surgery), gastrointestinal bleeding and minor bleeding. The quality of treatment with the use of warfarin was assessed using the Rosendaal method to determine the percentage of time that the INR was in the prespecified target range: $2.0-3.0$ (TTR). ${ }^{21}$ All outcome events were independently adjudicated by at least two independent investigators unaware of the treatment assignments. 
Statistical analysis

Baseline characteristics of patients from LA and non-LA countries were compared using t-test for continuous variables and continuity adjusted $\chi^{2}$ test for categorical variables. We developed Cox regression models to calculate HRs, CIs and $\mathrm{p}$ values for efficacy and safety outcomes to assess potential interaction between treatments and geographical regions. Differences in LA and non-LA mortality rates were not tested for statistical significance since it was not a priori main objective of this post hoc analysis and direct comparisons would lack statistical power to yield any major conclusion regarding mortality rates due to the different number of patients enrolled in the trial across various participating countries, which usually occur due to different timelines for site activation during the conduction of the trial. The Cox models were built after including terms for treatment, geographical region and treatment-by-region interaction. All analyses were performed using SAS software V.9.2 (SAS Institute, Cary, North Carolina, USA).

\section{RESULTS}

\section{Patient characteristics and management}

A total of 956 patients from Latin America and 17157 patients from non-LA countries were included in this analysis. The distribution of patients in LA countries was the following: Argentina $(n=530)$, Brazil $(n=249)$, Colombia $(n=13)$, Mexico $(n=84)$ and Peru $(n=80)$. As compared with non-LA patients, patients from LA had significantly $(\mathrm{p}<0.01)$ higher proportions of permanent AF (70.7\% vs 33.2\%), prior heart failure $(41.1 \%$ vs $31.5 \%)$ and hypertension $(82.3 \%$ vs $78.7 \%)$; however, age $(71.6$ vs 71.5$)$, prior stroke $(11.5 \%$ vs $12.6 \%)$, $\mathrm{CHA}_{2} \mathrm{DS}_{2}\left(2.2\right.$ vs 2.1) and $\mathrm{CHA}_{2} \mathrm{DS}_{2}-\mathrm{VASc}$ (3.5 vs 3.6) scores were not different. At randomisation, VKA use was lower in LA patients as compared with non-LA patients (44\% vs 63\%; $\mathrm{p}<0.001)$; by contrast, prior aspirin use (48.4\% vs $39.1 \%$; $\mathrm{p}<0.001)$ and prior ACE inhibitor use (55.9\% vs $44.2 \% ; \mathrm{p}<0.001)$ were more frequent among LA patients versus non-LA patients, respectively. Creatinine clearance was lower among LA patients $(63.1 \mathrm{~mL} /$ $\min$ vs $73.3 \mathrm{~mL} / \mathrm{min}$; $\mathrm{p}<0.001)$. Baseline characteristics are shown as online supplementary table. Moreover, the average TTR in patients on warfarin during the trial was lower in LA $(n=304)$ than in non-LA $(n=5487)$ patients $(61.3 \% \pm 22.6 \%$ vs $64.6 \% \pm 19.6 \% ; \mathrm{p}=0.015$, respectively).

\section{Efficacy outcomes}

The annual rates of the primary endpoint stroke/SE were $1.47 \%$ in LA patients versus $1.45 \%$ in non-LA patients. Their annual stroke/SE rates were $0.91 \%$ versus $1.68 \%$ for dabigatran $150 \mathrm{mg}$ twice daily versus warfarin, respectively (HR $0.54 ; 95 \%$ CI 0.18 to 1.62 ). The annual stroke/ SE rates for dabigatran $110 \mathrm{mg}$ twice daily versus warfarin were 1.82 vs 1.68 , also not significantly different (HR 1.09; $95 \%$ CI 0.44 to 2.67 ) (table 1 and figure 1 ). With regards to death from any cause, HRs versus warfarin for dabigatran 110 and 150 doses were not different (0.98 LA and 0.90 non-LA, and 0.85 and 0.88 , respectively). Annual mortality rates were higher $5.81 \%$ vs $3.74 \%$ among LA patients relative to non-LA patients, respectively. There were no treatment-by-region interactions for either dose of DE on all efficacy outcomes.

\section{Safety outcomes}

The annual rates of major bleeding were $2.63 \%$ in LA patients versus $3.28 \%$ in non-LA patients. In LA patients, the annual rates of major bleeding were $2.01 \%$ (dabigatran $110 \mathrm{mg}$ twice daily) and 2.72\% (dabigatran $150 \mathrm{mg}$ twice daily), with relative risk reductions of $37 \%$ and $14 \%$ versus warfarin, respectively (figure 2 and table 2). In LA patients, life-threatening and total bleeding events were $31 \%$ and $24 \%$ lower with dabigatran $150 \mathrm{mg}$ twice daily compared with warfarin, and $17 \%$ and $32 \%$ lower with dabigatran etexilate $110 \mathrm{mg}$ twice daily compared with warfarin. ICH was $81 \%$ lower (nominally) with dabigatran $110 \mathrm{mg}$ twice daily than warfarin while no LA patient on dabigatran $150 \mathrm{mg}$ twice daily experienced an ICH (figure 2). There were no treatment-by-region interactions for either dose of dabigatran etexilate on safety outcomes.

\section{DISCUSSION \\ Efficacy and safety of dabigatran versus warfarin}

The key findings of this analysis of RE-LY are the following. First, the efficacy and safety benefits of dabigatran in LA and non-LA patients seem consistent with those shown in the RE-LY trial overall. Second, all-cause mortality was nominally around $50 \%$ higher among LA patients relative to non-LA patients. Third, TTR was lower in LA patients on warfarin therapy in the trial.

In terms of the study population clinical profile, there were some remarkable differences in the baseline characteristics, medical history and prior use of medications between LA and non-LA patients. VKA use at randomisation was lower in LA patients compared with non-LA counterparts. Moreover, a greater proportion of LA patients had hypertension, heart failure and lower creatinine clearance, which are important clinical variables that might impact on mortality and stroke risks. Moreover, permanent AF was more common among LA patients compared with non-LA patients, based on standardised definitions of type of AF. Indeed, data from the global RE-LY AF registry have shown that the greatest proportions of patients with permanent AF in emergency department settings were found in developing countries, that is, $81.4 \%$ in Africa, $54.8 \%$ in China, $52.3 \%$ in Eastern Europe and $53.4 \%$ in Brazil. ${ }^{12}$ However, only a minority of those patients with $\mathrm{AF}$ are receiving recommended treatment with warfarin $(24.3 \%$ in South America, ranging from $13.1 \%$ in China to $42.6 \%$ in North America), thus reflecting significant variations in daily practice across geographical regions. The higher incidence of stroke or SE observed in LA patients in 


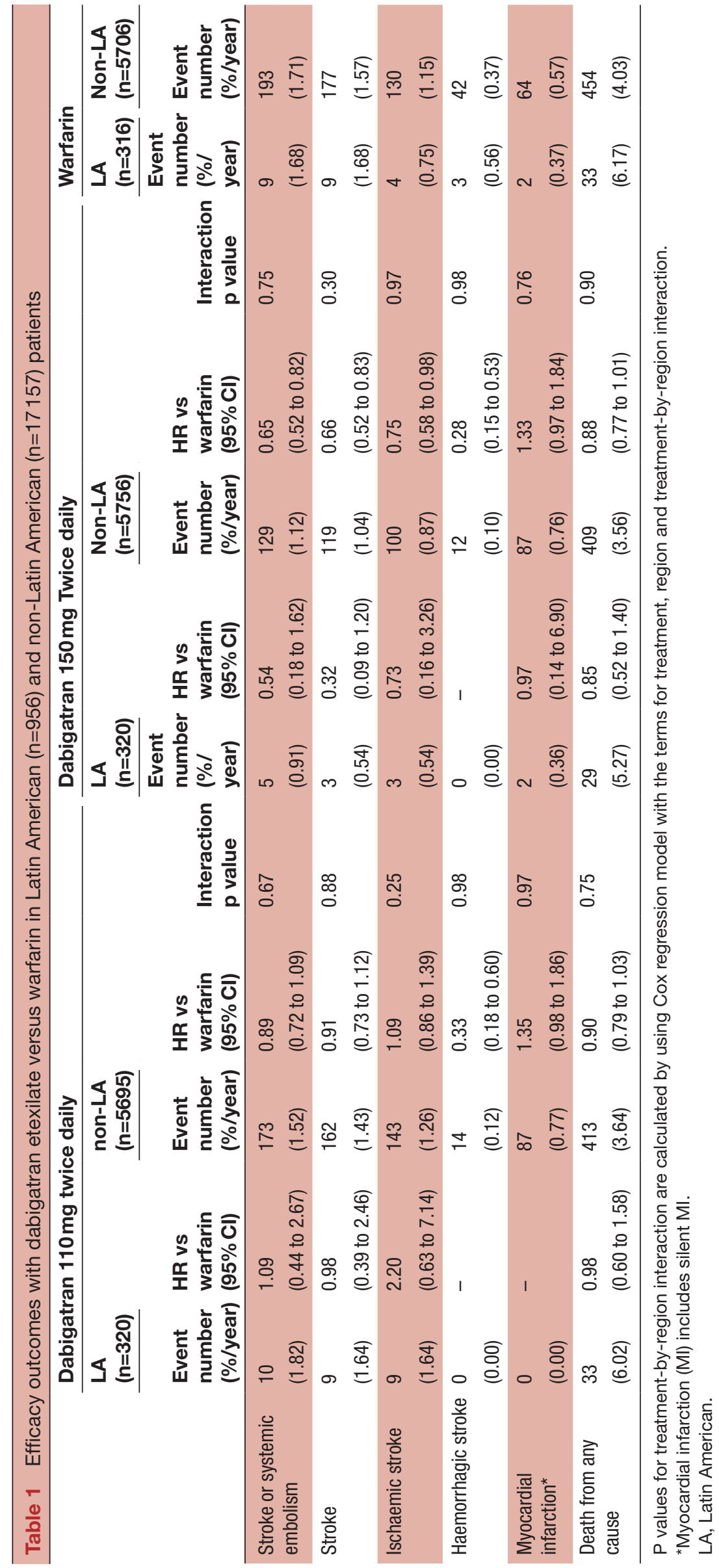




\section{Stroke or SE}

Latin America

Non-Latin America

Stroke

Latin America

Non-Latin America

Haemorrhagic stroke

Latin America

Non-Latin America

Myocardial infarction

Latin America

Non-Latin America

Death from any cause

Latin America

Non-Latin America

Ischaemic stroke

Latin America

Non-Latin America

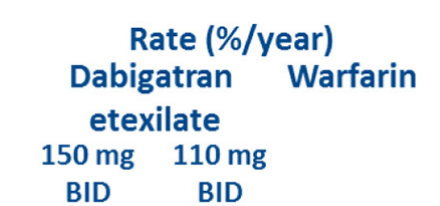

Dabigatran etexilate

$150 \mathrm{mg}$ BID

vs. warfarin

BID BID

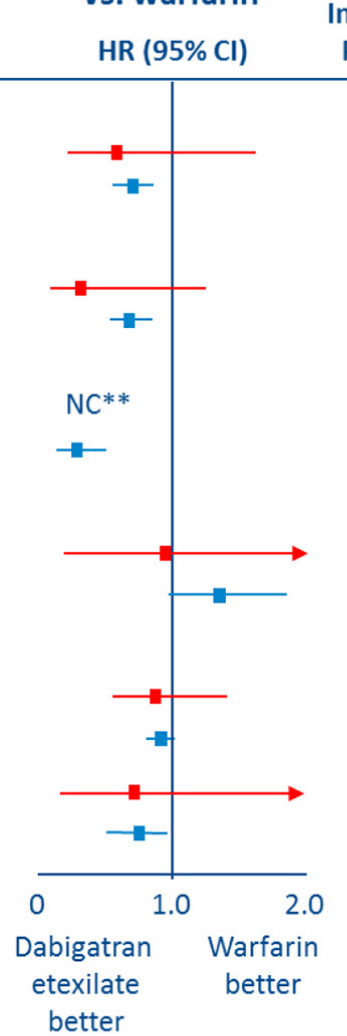

Dabigatran etexilate

$110 \mathrm{mg}$ BID

vs. warfarin

Interaction

Interaction combination with lower use of VKA enhances current knowledge of a large gap in appropriate management of care in AF setting, thus leading to great opportunity for improvement in adequate delivery of guideline-recommended AF management of care, that is, offering appropriate anticoagulation therapy when clinically indicated; increasing access to anticoagulation options of effective treatments and follow-up facilities.

The global variations in TTR and stroke risk factors aforementioned also might explain, at least in some degree, the results observed in this analysis, particularly the higher mortality risk among LA patients relative to non-LA patients. Recently developed clinical risk prediction models have also been derived from and validated in broad geographical regions, including patients from LA. ${ }^{22}$

Due to the lack of statistically significant interaction between geographical region and treatment effect, our data suggest the benefit and safety of dabigatran in comparison with warfarin for preventing stroke/SE in LA patients is consistent with the result observed in the main RE-LY trial and reassures the consistent findings from other subanalyses from other middle-income and low-income countries, such as Asian populations. ${ }^{18} 19$

Furthermore, we observed a lower average TTR among warfarin patients from Latin America than among their non-LA counterparts in the trial $(61 \%$ vs $65 \%, \mathrm{p}=0.015$, respectively). More importantly, when we take the realworld data into consideration, such as the RE-LY AF registry, ${ }^{12}$ the mean TTR was $46.8 \%$ in South America, ranging from $32.7 \%$ in Africa to $62.4 \%$ in Western Europe, with significant variations across geographical regions. In fact, since the desirable target of high quality of long-term anticoagulation with well-managed warfarin use has not been widely achieved, our findings are relevant for the real-world applicability and effectiveness of NOACs.

Subgroups of AF patients from LA are at increased risk of recurrent stroke compared with non-Hispanic whites. ${ }^{6} 79$ Possible reasons that could explain this increased risk are not fully understood. Although similar $\mathrm{CHA}_{2} \mathrm{DS}_{2}$ and $\mathrm{CHA}_{2} \mathrm{DS}_{2}$-VASc scores were observed between the two geographical regions, there were greater proportions of hypertension, heart failure and impaired renal function and higher annual rates of death from any 


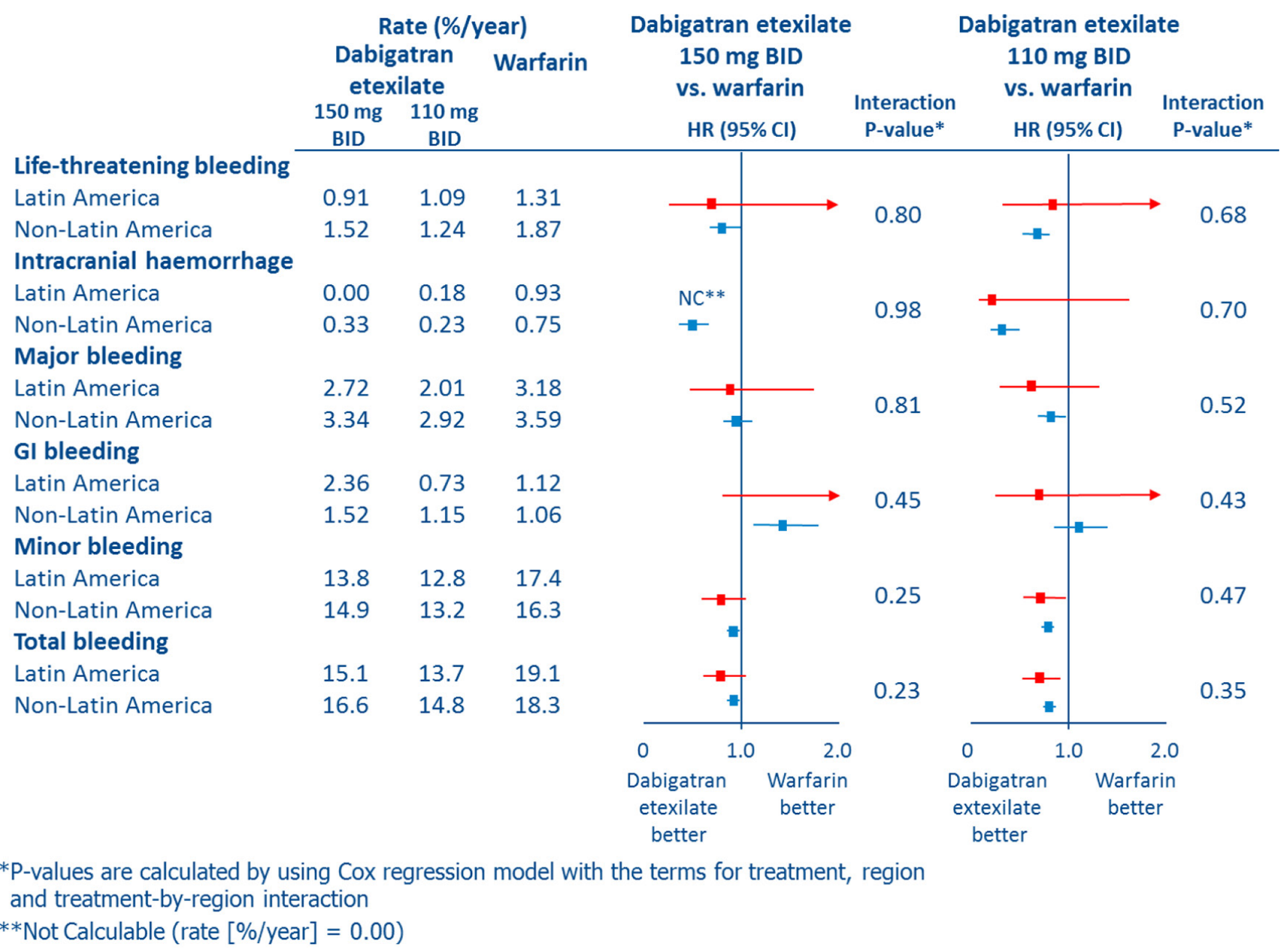

Figure 2 Safety outcomes with dabigatran etexilate versus warfarin in Latin American ( $\mathrm{n}=956)$ and non-Latin American $(n=17157)$ patients. BID, twice daily; Gl, gastrointestinal; NC, not calculable.

cause. Furthermore, the suboptimal use of anticoagulant therapies aforementioned and the lack of ideal TTR reflecting inadequate management of VKAs in South America and specific subgroups are likely to be contributing factors. ${ }^{10-12}$

The use and effectiveness of warfarin varies significantly depending on geographical region. Among patients with $\mathrm{CHA}_{2} \mathrm{DS}_{2}$ score $\geq 2$, two-thirds receive appropriate antithrombotic treatment in the Middle East/Africa, while this proportion drops to $55 \%$ in Europe, $44 \%$ in Latin America and $32 \%$ in Asia. ${ }^{23}$

Finally, in our study, the rate of ICH was considerably lower in LA patients treated with dabigatran versus warfarin (ie, nominally lower rates of $\mathrm{ICH}$ ) as observed in the main RE-LY trial. Since Hispanics have a significantly greater risk of ICH than non-Hispanic whites, ${ }^{7}$ reducing the risk of ICH in this population would be of greatest benefit at the population level.

\section{Interpretation}

The results of this analysis adds contemporary data on clinical characteristics, management and outcomes of patients with non-valvular AF from developing countries, especially LA, emphasising key practical features: consistency of effects regardless of geographical region; more favourable efficacy and safety profile with dabigatran; and large gap in AF clinical management thus leading to great room for improvement in the prevention of stroke and/or SE among LA populations, particularly after taking into account the striking higher mortality in LA patients.

There were some differences in terms of clinical characteristics, medical history and prior use of treatments between the overall and the LA populations; however, there were no significant differences in age, prior stroke or the mean $\mathrm{CHA}_{2} \mathrm{DS}_{2}$ or $\mathrm{CHA}_{2} \mathrm{DS}_{2}$-VASc score points. The majority of LA patients had permanent AF $(70.7 \%)$, while there was a roughly equal distribution of patients with permanent, paroxysmal and persistent $\mathrm{AF}$ in the non-LA cohort. Possible explanations for this finding include less frequent diagnosis of other types of $\mathrm{AF}$ and/ or selection bias due to more frequent screening among permanent patients with $\mathrm{AF}$ with clinical evaluation, echocardiograms, liver and renal function and coagulation tests to assess eligibility for long-term anticoagulation therapy. In addition, different physician perceptions of stroke risk among various types of AF may play a role. 


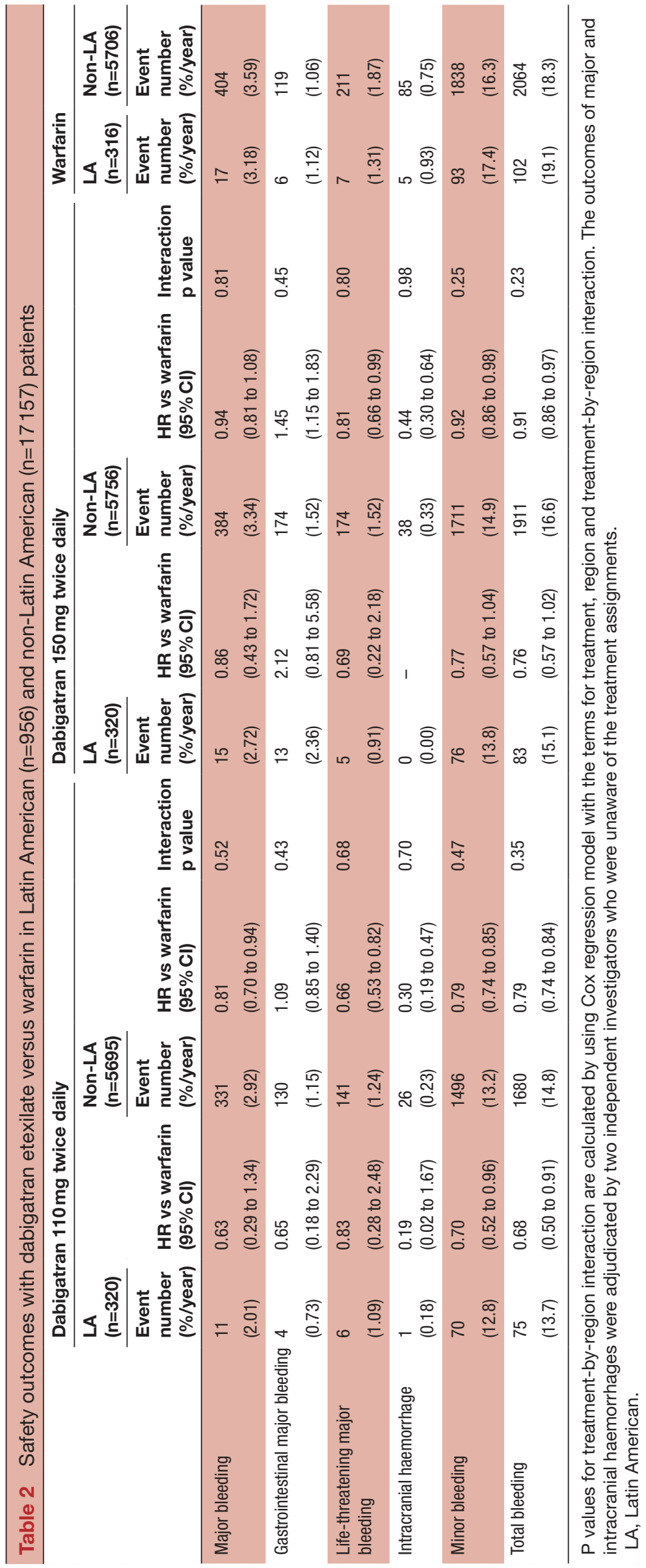


The use of VKAs at randomisation was significantly lower in LA patients compared with non-LA patients. This is a similar trend to that seen in a previous RE-LY subanalysis of Asian (36.5\%) versus non-Asian (52.0\%) patients. The number of patients with previous myocardial infarction (MI) was significantly lower in LA patients $(8.9 \%)$ versus non-LA patients $(17.0 \%)$. This is again similar to the trend seen in previous RE-LY subanalyses; the incidence of prior MI in Asian (9.3\%) and Japanese (5.5\%) patients was considerably lower compared with non-Asian patients (17.9\%) and the overall RE-LY population (16.6\%), respectively ${ }^{18} 19$

Finally, the assessment of the mean time in TTR indicates less effective management of care with warfarin therapy in LA patients, thus leading to greatest benefit to be derived from NOACs and, in addition, stronger systems for administering and monitoring VKA use in daily practice also are warranted to improve the quality of care in terms of sustained oral anticoagulation.

\section{Study strengths and limitations}

This subgroup analysis was not prespecified and there may be lack of statistical power to reliably detect differences in efficacy and safety with dabigatran versus warfarin. Nevertheless, the efficacy and safety benefits observed in the overall RE-LY trial were consistent between LA and non-LA patients, irrespective of the differences in baseline characteristics and numerical variations in the annual rates of the efficacy and safety endpoints, as compared with those seen in the entire trial population. Despite the open-label allocation of treatments in the RE-LY, all the efficacy and safety endpoints used for this analysis were ascertained during the trial and adjudicated by an independent adjudication committee who was unaware of the treatment assigned and followed standardised definition criteria. One limitation of this report was the relatively small number of LA patients $(n=956)$ compared with non-LA counterparts ( $\mathrm{n}=17157)$, as already discussed in this manuscript. Other aspects to be pointed out herein include the fact that patients enrolled in randomised clinical trials usually are highly selected, especially in trials of oral anticoagulation, that is, screening for eligibility clinical criteria to be enrolled in the trial must be rigorously performed and various contraindications to receive any oral anticoagulant must be assessed, which might limit external validation (generalisability of results). In contrast, patients not selected from randomised trials ('real world practice') have more comorbidities and relatively higher risk as compared with those recruited from randomised clinical trials.

\section{CONCLUSIONS}

Despite significant differences in the clinical profiles and long-term management of care for AF between LA and non-LA patients in the RE-LY trial, the efficacy and safety benefits of dabigatran over warfarin seem consistent with those observed in the main RE-LY trial. Coupled with significant higher mortality and a lower achieved TTR indicating less effective management of care with warfarin therapy in LA patients, our data suggest that the clinical benefit from implementation of non-VKA oral anticoagulants might be of greatest benefit as compared with warfarin.

Contributors The authors were involved at all stages of manuscript development. Funding The RE-LY studies were funded by Boehringer Ingelheim.

Competing interests None declared.

Patient consent Not required.

Ethics approval Institutional Review Board, Population Health Research Institute, Hamilton, Canada.

Provenance and peer review Not commissioned; externally peer reviewed.

Data sharing statement No additional data are available.

Open access This is an open access article distributed in accordance with the Creative Commons Attribution Non Commercial (CC BY-NC 4.0) license, which permits others to distribute, remix, adapt, build upon this work non-commercially, and license their derivative works on different terms, provided the original work is properly cited and the use is non-commercial. See: http://creativecommons.org/ licenses/by-nc/4.0/

(c) Article author(s) (or their employer(s) unless otherwise stated in the text of the article) 2018. All rights reserved. No commercial use is permitted unless otherwise expressly granted.

\section{REFERENCES}

1. Wolf PA, Abbott RD, Kannel WB. Atrial fibrillation as an independent risk factor for stroke: the Framingham Study. Stroke 1991;22:983-8.

2. StopAfib.org. Stroke risks from Afib. http://www.stopafib.org/stroke. cfm (Last accessed Sep 2013).

3. Go AS, Hylek EM, Phillips KA, et al. Prevalence of diagnosed atrial fibrillation in adults: national implications for rhythm management and stroke prevention: the AnTicoagulation and Risk Factors in Atrial Fibrillation (ATRIA) Study. JAMA 2001;285:2370-5.

4. Lip GYH, Brechin CM, Lane DA. The global burden of atrial fibrillation and stroke: a systematic review of the epidemiology of atrial fibrillation in regions outside North America and Europe. Chest 2012;142:1489-98.

5. Zimerman LI, Fenelon G, Martinelli Filho M, et al. Sociedade brasileira de cardiologia diretrizes brasileiras de fibrilação atrial. Arq Bras Cardiol 2009;92(Supp 1):1-39.

6. Simpson JR, Zahuranec DB, Lisabeth LD, et al. Mexican Americans with atrial fibrillation have more recurrent strokes than do nonHispanic whites. Stroke 2010;41:2132-6.

7. Shen AY, Yao JF, Brar SS, et al. Racial/ethnic differences in the risk of intracranial hemorrhage among patients with atrial fibrillation. $J$ Am Coll Cardiol 2007;50:309-15.

8. Camargo EC, Bacheschi LA, Massaro AR. Stroke in Latin America. Neuroimaging Clin N Am 2005;15:283-96.

9. Morgenstern LB, Smith MA, Lisabeth LD, et al. Excess stroke in Mexican Americans compared with non-Hispanic whites: the Brain Attack Surveillance in Corpus Christi Project. Am J Epidemiol 2004;160:376-83.

10. Birman-Deych E, Radford MJ, Nilasena DS, et al. Use and effectiveness of warfarin in Medicare beneficiaries with atrial fibrillation. Stroke 2006;37:1070-4.

11. Leiria TL, Pellanda L, Miglioranza MH, et al. [Warfarin and phenprocoumon: experience of an outpatient anticoagulation clinic]. Arq Bras Cardiol 2010;94:41-5.

12. Oldgren J, Healey JS, Ezekowitz M, et al. Variations in cause and management of atrial fibrillation in a prospective registry of 15,400 emergency department patients in 46 countries: the RE-LY Atrial Fibrillation Registry. Circulation 2014;129:1568-76.

13. Costa GL, Ferreira DC, Valacio RA, et al. Quality of management of oral anticoagulation as assessed by time in therapeutic INR range in elderly and younger patients with low mean years of formal education: a prospective cohort study. Age Ageing 2011;40:375-81.

14. Connolly SJ, Ezekowitz MD, Yusuf S, et al. Dabigatran versus warfarin in patients with atrial fibrillation. $N$ Engl J Med 2009;361:1139-51. 
15. Granger CB, Alexander JH, McMurray JJ, et al. Apixaban versus warfarin in patients with atrial fibrillation. $N$ Engl J Med 2011;365:981-92.

16. Patel MR, Mahaffey KW, Garg J, et al. Rivaroxaban versus warfarin in nonvalvular atrial fibrillation. N Engl J Med 2011;365:883-91.

17. Giugliano RP, Ruff CT, Braunwald E, et al. Edoxaban versus warfarin in patients with atrial fibrillation. N Engl J Med 2013;369:2093-104.

18. Hori M, Connolly SJ, Ezekowitz MD, et al. Efficacy and safety of dabigatran vs. warfarin in patients with atrial fibrillationsub-analysis in Japanese population in RE-LY trial. Circ J 2011;75:800-5.

19. Hori M, Connolly SJ, Zhu J, et al. Dabigatran versus warfarin: effects on ischemic and hemorrhagic strokes and bleeding in Asians and non-Asians with atrial fibrillation. Stroke 2013;44:1891-6.
20. Ezekowitz MD, Connolly S, Parekh A, et al. Rationale and design of RE-LY: randomized evaluation of long-term anticoagulant therapy, warfarin, compared with dabigatran. Am Heart J 2009;157:805-10.

21. Rosendaal FR, Cannegieter SC, van der Meer FJ, et al. A method to determine the optimal intensity of oral anticoagulant therapy. Thromb Haemost 1993;69:236-9.

22. Hijazi Z, Oldgren J, Lindbäck J, et al. A biomarker-based risk score to predict death in patients with atrial fibrillation: the ABC (age, biomarkers, clinical history) death risk score. Eur Heart $J$ 2018;39:477-85.

23. Gamra H, Murin J, Chiang CE, et al. Use of antithrombotics in atrial fibrillation in Africa, Europe, Asia and South America: insights from the International RealiseAF Survey. Arch Cardiovasc Dis 2014;107:77-87. 